\title{
is Research Soure \\ Modulation of Actin network and Tau phosphorylation by HDAC6 ZnF UBP domain
}

\author{
Abhishek Ankur Balmik \\ National Chemical Laboratory CSIR \\ Shweta Kishor Sonawane \\ National Chemical Laboratory CSIR \\ Subashchandrabose Chinnathambi ( $\nabla$ s.chinnathambi@ncl.res.in ) \\ National Chemical Laboratory CSIR https://orcid.org/0000-0002-5468-2129
}

\section{Research}

Keywords: HDAC6, Tau, phosphorylation, Tauopathies, microtubule, actin, podosomes, podonut, actin, cytoskeleton, neurodegeneration

Posted Date: February 13th, 2020

DOI: https://doi.org/10.21203/rs.2.23349/v1

License: (c) (i) This work is licensed under a Creative Commons Attribution 4.0 International License. Read Full License 


\section{Abstract}

Background: Microtubule-associated protein Tau undergoes aggregation in Alzheimer`s disease and a group of other related diseases collectively known as Tauopathies. In AD, Tau forms aggregates, which are deposited intracellularly as neurofibrillary tangles. HDAC6 plays an important role in aggresome formation where it recruits poly-ubiquitinated aggregates to the motor protein dynein. Methods: Here, we have studied the effect of HDAC6 ZnF UBP on Tau phosphorylation, ApoE localization, GSK-3ß regulation and cytoskeletal organization in neuronal cells by immunofluorescence analysis using fluorescently tagged molecules or antibodies. We have assessed neuronal cell viability and membrane integrity under different treatment conditions by employing MTT and LDH assay respectively. Results: Immunocytochemistry reveals that HDAC6 ZnF UBP can modulate Tau phosphorylation and actin cytoskeleton organization when the cells are exposed to the domain. HDAC6 ZnF UBP treatment to cells does not affect their viability and resulted in enhanced neurite extension and formation of structures similar to podosomes, lamellipodia and podonuts suggesting its role in actin re-organization. Also, HDAC6 treatment showed increased nuclear localization of ApoE and tubulin localization in microtubule organizing centre. Conclusions: Altogether, our studies suggest the regulatory role of this domain in different aspects related to neurodegenerative diseases.

\section{Introduction}

Tau pathology is implicated in several neurodegenerative diseases including Alzheimer's and Parkinson's disease, Progressive supranuclear palsy, Pick's disease, Corticobasal degeneration and Post-encephalitic parkinsonism [1, 2]. Tau is comprised of two $\mathrm{N}$-terminal inserts, a poly-proline domain and four imperfect repeat domains (Fig. 1A). The four repeat regions are the functional and pathological unit of Tau as it is involved in microtubule-binding as well as aggregate formation. In Alzheimer's disease and related Tauopathies, Tau gets detached from microtubules and associates with other Tau molecules to form intracellular aggregates in the form of NFTs $[3,4]$. Tau acquires pathological form either through the genetic predisposition on chromosome 17 termed as FTDP-17 or hyperphosphorylation and several other post-translational modifications $[5,6]$. Phosphorylation is a well-studied PTM of Tau responsible for its conversion to pathological form in $A D$ condition [7-9]. GSK-3 $\beta$ is one of the major kinase involved in Tauopathies [10]. Tau is phosphorylated by GSK-3ß on both primed (after pre-phosphorylation) and unprimed sites and affects its ability to bind and stabilize microtubules [11, 12].In Alzheimer's disease, the structure and function of Tau protein is modified by various cellular and molecular factors such as oxidative stress, post-translational modifications and interaction with its binding partners [13]. This result in the accumulation of Tau protein aggregates as intracellular inclusion bodies called neurofibrillary tangles (NFTs). The abnormal accumulation of Tau as NFTs affects various cellular processes including microtubule dynamics and intracellular transport mechanism dependent on the cytoskeletal networks.

The proteins upon misfolding are targeted for degradation by ubiquitination and these poly-ubiquitinated protein aggregates are degraded and recycled in proteasome [14-17]. Aggregation and subsequent accumulation of Tau protein aggregates are primarily tackled by chaperones and ubiquitin proteasomal 
system. In case of disruption of ubiquitin proteasome system (UPS), the aggregates are directed towards the formation of aggresomes which serves as the cytoprotective response upon the failure of UPS [1720]. HDAC6 is a class II histone deacetylase mainly present in the cytoplasm involved in the regulation of various cellular functions. It consists of two catalytic deacetylase domains and a unique ZnF UBP (Zinc finger ubiquitin binding protein) domain, which sets it apart from other HDACs [21-23]. The proteins upon misfolding are targeted for degradation by ubiquitination and these poly-ubiquitinated protein aggregates are degraded and recycled in proteasome [14-17]. Aggregation and subsequent accumulation of Tau protein aggregates are primarily tackled by chaperones and ubiquitin proteasomal system. In case of disruption of ubiquitin proteasome system (UPS), the aggregates are directed towards the formation of aggresomes which serves as the cytoprotective response upon the failure of UPS [1720]. One of the major functions of HDAC6 is recruiting the polyubiquitinated protein aggregates to Dynein/Dynactin complex and carrying them to MTOC for aggresome formation. HDAC6 acts on cortactin and mediates its association with F-actin to facilitate cell motility [24]. The function of HDAC6 in both UPS and autophagy indicate its role as a possible link between the two mechanisms [25-27]. The impairment of UPS function acts as a cue for the activation of a compensatory mechanism for the clearance of protein aggregates. It has been studied in the Drosophilla model of spinobulbar muscular atrophy, where expression of HDAC6 has been found to effectively cause rescue from UPS impairment induced neurodegeneration by triggering the autophagic clearance of protein aggregates [26]. In another study, HeLa cells transfected with PolyQ Huntingtin forms intracellular protein aggregates, which require HDAC6 for autophagic clearance after inhibition of proteasomal system [28]. Overall, the function of HDAC6 with respect to protein aggregate clearance and autophagy induction serves as a protective mechanism. The HDAC6 expression level increases sharply in protein misfolding diseases [29].

Tau exists in hyperphosphorylated state in NFTs. Alzheimer's disease is associated with the upregulation of cellular kinases like GSK-3 $\beta$ and CDK5 [30]. There a number of serine and threonine residues in repeat region of Tau which are phosphorylated in $A D$ through proline directed kinases [31]. On the other hand protein phosphatases like PP1 and PP2A are known to be downregulated in AD failing to reverse the effect of hyperphosphorylation [32-34]. Thus, there is an imbalance between the kinase and phosphatase function in neurons. Another important aspect of neurodegenerative diseases involve the mis-functioning of its cytoskeletal elements. Microtubule network and actin organization were found to be distorted leading to impaired cellular trafficking and other associated functions [35, 36]. Actin organization is crucial for synaptic signalling in neurons where they are involved in the formation of dendritic spines for neurotransmission. Impaired actin assembly and depolymerisation leads to loss of dendritic spines ultimately causing neuronal death [37, 38].HDAC6 is a key protein in the regulation of both actin and microtubule organization through its deacetylase activity [39]. However, the role of its $\mathrm{ZnF}$ UBP domain in cytoskeletal function is lesser studied.In the present study, we studied the role of HDAC6 ZnF UBP domain (Fig. 1B) in modulating different cellular events such as phosphorylation, cytoskeletal assembly and cellular localization. HDAC6 ZnF UBP domain was found to affect actin and tubulin organization, Tau phosphorylation and localization of ApoE in neuronal cells. Enhancement in podosome and lamellipodia-like structures were found when neuronal cells were exposed to HDAC6 ZnF UBP 
domain suggesting its direct role in actin organization. HDAC6 ZnF UBP treatment also resulted in enhanced tubulin localization in MTOC indicating its possible role in tubulin polymerization events. Our findings suggest the role of HDAC6 ZnF UBP as the direct modulator of Tau phosphorylation and cytoskeletal dynamics.

\section{Materials And Methods}

\section{Chemicals and reagents}

Luria-Bertani broth (Himedia); Ampicillin, $\mathrm{NaCl}$, Phenylmethylsulfonylfluoride (PMSF), $\mathrm{MgCl}_{2}$, APS, DMSO, Ethanol (Mol Bio grade), Isopropanol (Mol Bio grade) were purchased from MP biomedicals; IPTG and Dithiothreitol (DTT) from Calbiochem; MES, BES, SDS from Sigma; EGTA, Protease inhibitor cocktail, Tris base, 40\% Acrylamide, TEMED from Invitrogen. For cell culture studies, Dulbecco modified eagle's media (DMEM), Fetal bovine Serum (FBS), Horse serum, Phosphate buffer saline (PBS, cell biology grade), Trypsin-EDTA, Penicillin-streptomycin, Pierce ${ }^{\text {TM }}$ LDH Cytotoxicity Assay Kit (Thermo, cat no 88953), RIPA buffer were also purchased from Invitrogen. MTT reagent, Okadaic acid and TritonX-100 were purchased from Sigma. The coverslip of $0.17 \mathrm{~mm}$ was purchased from Bluestar for immunofluorescence. In immunofluorescence and western blot study we used the following antibodies: Beta-actin (Thermofisher cat no. MA515739) Beta Tubulin (BT7R) (Thermofisher, cat no MA516308) and total Tau antibody K9JA (Dako, cat no A0024), pT181 (Invitrogen, cat no 701530) AT8 (Thermo fisher, cat no MN1020), GSK-3 $\beta$ (Thermo fisher, cat no MA5-15109), Phospho-GSK-3ß (Ser9) (Thermo fisher, cat no MA5-14873), antiApoE (Sigma, cat no. SAB2701946), anti-mouse secondary antibody conjugated with Alexa Fluor-488 (Invitrogen, cat no A-11001), Goat anti-Rabbit IgG $(\mathrm{H}+\mathrm{L})$ Cross-Adsorbed Secondary Antibody with Alexa Fluor 555 (A-21428), Rabbit anti-Goat IgG (H+L) Cross-Adsorbed Secondary Antibody with Alexa Fluor 594 (A27016) and DAPI (Invitrogen).

\section{Protein expression and Purification}

Full length Tau (hTau40wt) in pT7C were transformed and expressed in BL21* cells while HDAC6 in pET28a-LIC was transformed and expressed in BL21 Codon plus RIL cells. Full-length Tau and repeat domain Tau were purified in two steps using cation exchange chromatography and Size-exclusion chromatography. The cells expressing these proteins after transformation were scaled up and harvested. The cells were lysed by homogenization at $15000 \mathrm{KPSI}$. The lysate was supplied with $0.5 \mathrm{M} \mathrm{NaCl}$ and 5 $\mathrm{mM}$ DTT and kept at $90^{\circ} \mathrm{C}$ for 20 minutes to denature all the structured protein. The resulting sample was centrifuged at $40000 \mathrm{rpm}$ for 45 minutes. The supernatant was kept for overnight dialysis in $20 \mathrm{mM}$ MES $\mathrm{pH}$ 6.8. The dialyzed sample was centrifuged again at $40000 \mathrm{rpm}$ for 45 minutes and the supernatant was filtered and loaded onto Sepharose fast flow (SPFF) column pre-equilibrated with $20 \mathrm{mM}$ MES pH $6.8,50 \mathrm{mM} \mathrm{NaCl}$. Elution was carried out using $20 \mathrm{mM} \mathrm{MES} \mathrm{pH} \mathrm{6.8,1} \mathrm{M} \mathrm{NaCl}$. The fractions collected from cation exchange chromatography containing Tau protein were pooled, concentrated and subjected to Size-exclusion chromatography using 1X PBS, 2 mM DTT in Superdex 75 Hi-load 16/600 column [72, 73]. Purification of HDAC6 ZnF UBP was carried out by Ni-NTA affinity chromatography using $50 \mathrm{mM}$ Tris- 
Cl pH 8.0 with 20 mM Imidazole for wash and 1000 mM imidazole for elution. The sample was dialyzed overnight in $50 \mathrm{mM}$ Tris-Cl pH 8.0, $100 \mathrm{mM} \mathrm{NaCl}, 2.5 \%$ glycerol to remove imidazole followed by Sizeexclusion chromatography using Superdex 75 Hi-load 16/600 column [63].

\section{Cell viability by MTT assay}

The effective concentration of HDAC6 for the subsequent treatments was determined by studying the concentration dependent toxicity studies by MTT assay. $10^{4}$ neuro2a cells (ATCC CCL-131) were seeded in a 96 well culture plate in DMEM supplemented with 10\% FBS and antibiotic penicillin-streptomycin for 24 hours at $37^{\circ} \mathrm{C} \mathrm{CO}_{2}$ incubator. The cells were treated with HDAC6 (0- $500 \mathrm{nM}$ ) in serum-starved media for 24 hours. MTT at the concentration of $0.5 \mathrm{mg} / \mathrm{mL}$ was added to the cells and incubated for 3 hours. The reduction of MTT by cellular enzymes forms formazon crystals, which were dissolved in DMSO, and the colour developed was quantified by reading at $570 \mathrm{~nm}$ in a TECAN Infinite 200 PRO plate reader.

\section{LDH assay}

The effect of HDAC6 treatment on cell membrane integrity was studied by LDH (Lactate Dehydrogenase) assay. The disruption of cell membrane integrity leads to the leakage of LDH enzyme, which is quantified by an enzymatic reaction giving a colored end product. For performing LDH assay the cells were incubated and treated as mentioned for MTT assay. After the treatment with HDAC 6 supernatant media was used and the assay was performed according to the manufacturer's protocol. In brief, $50 \mu \mathrm{L}$ of cell supernatant was incubated with $50 \mu \mathrm{L}$ of the reaction mixture provided for 30 minutes at room temperature. $50 \mu \mathrm{L}$ of stop solution was added to each well and the colour developed was measured at $490 \mathrm{~nm}$ and background subtraction at $680 \mathrm{~nm}$ was done.

\section{Caspase $3 / 7$ activity assay}

In order to study the effect of HDAC6 on inducing apoptotic cell death, the activity of executioner caspase 3 was determined by EnzChek ${ }^{\text {TM }}$ Caspase-3 Assay Kit. 10000 cells/well cells were seeded in a 12 well culture plate for 24 hours and further treated with HDAC6 (0-500 nM) for 24 hours in serum-starved media. Caspase activity was performed as per manufacturer's protocol. The cells were lysed with provided lysis buffer in freeze-thaw cycles. The cell debris was centrifuged out and the supernatant was incubated with fluorescent substrate (DEVD-Rhodamine). The fluorescence was quantified at (Ex/Em) 496 /520 nm at different time intervals in TECAN Infinite 200 PRO plate reader.

\section{Cell culture and Immunofluorescence}

Neuro2a cells were cultured in advanced DMEM supplemented with penstrep-glutamine, anti-mycotic and $10 \%$ FBS. For immunofluorescence studies $5 \times 10^{4}$ cells were seeded on a glass coverslip (Bluestar) in a 12 well culture plate. Cells were given the desired treatment in serum starved media (0.5\% FBS) for 24 hours including groups involving Okadaic acid (OA) treatment. After incubation period, cells were washed with PBS and fixed with $4 \%$ paraformaldehyde. Further cells were washed with PBS thrice and 
permiabilized using $0.2 \%$ Triton $X-100$. Cells were blocked with $2 \%$ horse serum and incubated with primary antibodies in a moist chamber at $4{ }^{\circ} \mathrm{C}$ overnight. Next day, cells were washed thrice with $1 \mathrm{X}$ PBS and incubated with alexa fluor labeled secondary antibodies for 1 hour at $37^{\circ} \mathrm{C}$. The unbound secondary antibody was washed off with three washes of PBS and counterstained with DAPI. The coverslips were mounted in $80 \%$ glycerol and observed under $63 \mathrm{X}$ oil immersion lens in Axio Observer 7.0 Apotome 2.0 (Zeiss) microscope.

\section{Statistical analysis}

Two-tailed unpaired student t-test was used to determine the significance for experiments involving

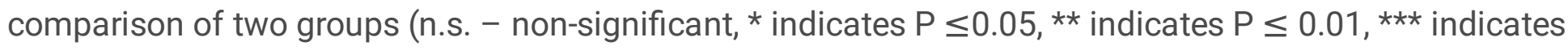
$P \leq 0.001$ ). One way ANOVA was conducted for the levels of phospho-epitopes (pT181 and AT8) to compare different treatment groups. Tukey's HSD (Honest significant difference) test was performed to compare the significance within groups (Significant at Tukey's HSD $p<0.05$ ). All the experiments were performed in triplicates and analyzed by Sigmaplot 10.0 (Systat software).

\section{Results}

\section{HDAC6 ZnF UBP is non-toxic and non-apoptotic}

Neuronal cells were exposed to HDAC6 ZnF UBP exogenously to observe the effect on different cellular functions. In order to study the effects of HDAC6 ZnF UBP, a minimum toxic dose was determined by viability assay as well as membrane leakage assays. Neuronal cells were treated with a range of concentrations of HDAC6 ZnF UBP (20-500 nM). The HDAC6 internalization was monitored for 20-500 nM concentrations by immunostaining for HDAC6 and anti-His-tag (Supp. Fig. 1). The cell viability carried out by MTT assay showed no decrease in viability. The cell viability was maintained at $80 \%$ even in highest HDAC6 ZnF UBP concentration of 500 nM (Fig.1C). Similarly, LDH assay was carried out to check the effect of HDAC6 on membrane integrity in terms of $L D H$ release. Neuronal cells showed intact membrane integrity when treated with HDAC6 ZnF UBP in 20-500 nM concentration range (Fig.1D). Thus, HDAC6 ZnF UBP did not show toxicity in neuroblastoma cells. Further to confirm the non-toxic nature of HDAC6 ZnF UBP in neuronal cells we studied the apoptosis on treatment with HDAC6 ZnF UBP by caspase- 3 assay. Cells undergo apoptosis under stressful conditions-mediated by endoproteases called caspases. Caspase- 3 is an executioner caspase responsible for DNA fragmentation and degradation of cytoplasmic proteins. The caspase- 3 assay showed basal level of activity in all the treated and control samples. No difference was observed in the HDAC6 ZnF UBP treated and untreated control samples in terms of cell viability, suggesting that HDAC6 ZnF UBP treatment do not induce apoptosis in the neuro2a cells (Fig.1E). The preliminary studies on cell viability and morphology inferred that HDAC6 ZnF UBP treatment do not show cytotoxic effect on neuro2a cells. Hence, the cells were treated with a moderate concentration of $50 \mathrm{nM}$ HDAC6 ZnF UBP for subsequent experiments.

\section{HDAC6 ZnF UBP enhances levels of pGSK-3 $\beta$}


Modulation of GSK-3 $\beta$ by HDAC6 ZnF UBP may involve regulation of Akt via PP1. PP1 dephosphorylates Akt which is a negative regulator of GSK-3 $\beta$ in its phosphorylated state [40]. HDAC6 is known to associate with PP1 through its C-terminal region which corresponds to ZnF UBP domain [41]. HDAC6 ZnF UBP addition to cells increases PP1-HDAC6 association rendering Akt in its active phosphorylated state, which in turn phosphorylates Ser9 on GSK-3 $\beta$ downregulating its activity (Fig. $2 A$ ). The level of GSK-3 $\beta$ in its phosphorylated and non-phosphorylated form determines its activity as a kinase. GSK-3 $\beta$ is known to associate with HDAC6 to counteract its function to induce LPS-tolerance in astrocytes [42]. We mapped GSK-3 $\beta$ and pGSK-3 $\beta$ (pSer9) levels by immunofluorescence in neuronal cells in basal conditions and upon HDAC6 ZnF UBP treatment. There was no marked difference in level of GSK-3 $\beta$ in HDAC6 treated and untreated cells (Fig.2B). However, immunofluorescence analysis of HDAC6 ZnF UBP treated cells showed significant increase in the pGSK-3 $\beta$ levels (Fig.2C). GSK-3 $\beta$ function in cells is regulated mainly by inhibitory phosphorylation at ser9 or ser 21 . The kinase activity of GSK-3 $\beta$ is reduced with phosphorylation at ser9 as it affects the binding of primed substrates with GSK-3 $\beta$. The increase in pGSK$3 \beta$ in neuronal cells signifies the reduced GSK-3 $\beta$ activity on primed Tau substrate [43]. HDAC6 ZnF UBP exposed to the cells resulted in increased levels of pGSK-3 $\beta$ (Ser9), which suggests reduced Tau

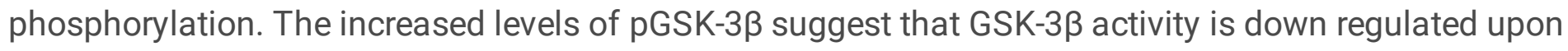
HDAC6 ZnF UBP treatment.

\section{HDAC6 ZnF UBP reduces Tau phosphorylation in neuronal cells}

Tau phosphorylation is the key event in the pathogenesis of Alzheimer's disease. Tau phosphorylation is required for its function in microtubule interaction and stabilization. Under pathological conditions Tau becomes hyperphosphorylated due to imbalance in kinase and phosphatase level or activity [7-9, 44] Okadaic acid (OA) was used as an inducer of hyperphosphorylation as it inhibits protein phosphatase $2 \mathrm{~A}$ (PP2A), thus increasing the overall phosphorylation level [45]. Untreated control cells and HDAC6 ZnF UBP treated cells alone showed basal levels of phospho-Tau at epitopes pT181 and pS202/T205 (AT8).

The levels of pT181 were increased in OA treated cells. Cells supplemented with HDAC6 ZnF UBP with OA showed lower levels of phospho Tau at T181 as compared to positive control (Fig.3A). Tau

phosphorylated at pT181 was dominantly seen in the nucleus especially in the positive control (enlarged images). Similar results were observed for phospho Tau epitope AT8. The enlarged images clearly show the increased levels of phospho-AT8 in positive control as compared to OA+HDAC6 ZnF UBP treatment (Fig.3B). pT181 and AT8 are two of the crucial epitopes of pathological Tau in AD. HDAC6 ZnF UBP treatment lowered down the level of these two phospho-epitopes suggesting its possible role in modulating Tau phosphorylation. Their immunofluorescence levels in different experimental groups (No quantified the level of both phospho-tau epitopes (pT181 and AT8). of fields selected for quantification of pT181 and AT8, $n=5)$. Untreated cells (CC) showed no significant difference from HDAC6 treated or OA+HDAC6 treated in pT181 immunostaining. OA treatment resulted in increased pT181 levels, which was found to be reduced with HDAC6 ZnF UBP treatment (Fig. 3C). Similar results were obtained for quantification of AT8 immunostaining. HDAC6 ZnF UBP treatment along with OA reduced AT8 level, bringing it down similar to untreated cells (CC) when compared to OA alone treatment group. AT8 levels were also found to be lesser in HDAC6 ZnF UBP treatment as compared to untreated (Fig. 3D). 
Quantification data was analyzed by one-way ANOVA followed by Tukey's HSD test (significant at $a=$ 0.05).

\section{HDAC6 ZnF UBP modulates actin dynamics}

Actin organization and dynamics is crucial for cell shape and migration in respective cell types. In neuronal cells, actin dynamics is important for cell-to-cell communication through regulating neurite extension. Actin assembly and dynamics requires complex machinery and nucleating factors [46, 47]. In neurodegenerative diseases, the regulation of actin dynamics gets impaired resulting in loss of synapses and dendritic spines [48]. HDAC6 is known to modulate actin organization through its association and deacetylase activity on cortactin and arp2/3 complex $[24,46,47]$. The function of HDAC6 ZnF UBP domain in actin dynamics is not yet understood. Our present observations showed HDAC6 ZnF UBP treatment lead to increase in neuritic extensions in neuro2a cells. We mapped HDAC6 ZnF UBP treated cells with FITC-Phalloidin to observe the F-actin cytoskeleton. We studied actin organization along with Tau and it was observed that HDAC6 ZnF UBP treatment had no effect on Tau levels but the actin cytoskeleton changed significantly (Fig.4A). Further, to check for HDAC6 localization on treatment, we mapped actin with HDAC6 ZnF UBP. The control cells showed a normal F-actin cytoskeleton in the extensions but HDAC6 treated cells showed presence of actin along with HDAC6 in these extensions (Fig. 4B). As previously observed, HDAC6 ZnF UBP treated cells showed more neuritic extensions and dense actin cytoskeleton. Tau and actin are known to co-localize in neuritic extensions in previous studies where they facilitate growth cone transition [49]. We also observed Tau-actin co-localization in neuritic extensions indicating the interplaying of microtubule and actin dynamics in formation of cell extensions (Supp. Fig. S2A, B). Greyscale images for actin immunostaining shows localization in neurite extensions and growth cones in filopodia-like structures (Fig. 4C, D).

\section{Enhancement of podosome-like structures by HDAC6 ZnF UBP}

Podosomes are actin based structures involved in the remodeling of extracellular matrix (ECM) and cell migration while podonuts consists of a cluster of podosomes interacting with ECM [50]. It consists of an actin rich core surrounded by actin regulatory molecules-like cortactin and arp $2 / 3$ as well as cell adhesion molecules like Talin and Vinculin [51]. Morphological changes in neuronal cells were observed when treated with HDAC6 ZnF UBP. HDAC6 ZnF UBP treated cells showed increased membrane ruffles and podosome-like structures (Supp. Fig. 3). Neuro2a cells were incubated with 50 nM HDAC6 ZnF UBP on 18 $\mathrm{mm}$ coverslips for 24 hours prior to immunostaining preparation. Immunostaining showed the localization of actin in the membrane ruffles in HDAC6 ZnF UBP treated cells. We observed minimal neuritic extensions in untreated neuro2a as compared to treated cells (Fig. 5A). Actin localization was observed mostly along the cell periphery in untreated neuro2a while it was focused in the neurite extensions and protruding podosome structure in HDAC6 ZnF UBP treated cells (Fig. 5B). Orthogonal sections were taken to clearly visualize the actin extensions and podosome formation. The initial orthogonal sections showed numerous extensions and actin localized in tip of the extensions in HDAC6 ZnF UBP treated cells as compared to control (Fig. 5C, D). Involvement of podosomes in cell adhesion 
and migration is an important attribute of invasive cells. Neuro2a, being a neuroblastoma cell line can form podosomes for cell migration and attachment. In contrast to untreated control neuronal cells (Fig.6A, B, C), HDAC6 ZnF UBP treated cells showed enhanced cell extensions and actin rich structures similar to podosomes formed in migrating and invading cells. HDAC6 treated cells showed a variety of actin-based membrane protrusions, which can be morphologically classified as filopodia or lamellipodia, podosomes and podonuts (Fig. 6D-J). The neuritic extensions and podosomes were quantified in untreated control cells (CC) and HDAC6 treated cells. The neurites and podosomes were counted manually in multiple fields for untreated control and HDAC6 treated group (No. of fields, $n=6$ ). HDAC6 ZnF UBP treated cells showed significantly enhanced neurite extensions and podosomes compared to untreated cells implicating its effect on actin dynamics and re-organization (Fig. 6K, L).

\section{HDAC6 ZnF UBP enhances ApoE nuclear localization}

ApoE functions mainly as a lipid carrier in CNS where it delivers cholesterol to neurons via ApoE receptors following neuronal injury [52]. ApoE can be localized to nucleus and carries out induction of gene expression involved in inflammatory response. ApoE is the major apolipoprotein of central nervous system, produced primarily by astrocytes. ApoE is taken up by neurons where it plays important role in membrane maintenance and repair [53]. In normal physiological conditions, ApoE is localized in cytosol. ApoE nuclear localization has been reported in ovarian cancer cells, where it leads to better survival possibly through gene regulation [54]. We treated neuro2a cells with $50 \mathrm{nM}$ of HDAC6 ZnF UBP domain to observe its effect on distribution and localization of ApoE in cell. It was observed that upon HDAC6 ZnF UBP treatment, there is increased nuclear localization of ApoE (Fig. 7A). The localization of ApoE was quantified by analyzing the intensity of ApoE immunofluorescence in nucleus and cytoplasm of untreated and HDAC6 ZnF UBP treated cells. The ApoE level in cytoplasm of both untreated and HDAC6 treated cells showed no change while it was significantly increased in the nucleus of HDAC6 ZnF UBP treated cells (Fig. 7B). The enhanced nuclear localization of ApoE may indicate in improved neuronal health [55]. ApoE can get translocated to the nucleus through a nuclear targeting chaperone nucleolin [54]. ApoE consists of weak nuclear localizing sequence, which indicates that there must be other mechanism involved in ApoE nuclear transport. Nucleolin is known to associate with apolipoproteins facilitating their translocation [56]. HDAC6 may mediate ApoE translocation through HSP90 as it is known to stabilize nucleolin during mitosis [57]. However, the exact mechanism needs to be further elucidated (Fig. 7C).

\section{Enhanced Tubulin localization to MTOC with HDAC6 ZnF UBP treatment}

[HDAC6 is a known interacting partner of tubulin and regulates microtubule structure and function through deacetylation. In a previous study, it was found that HDAC6 knockdown in cells along with HDAC6 inhibitor tubacin treatment does not affect microtubule growth velocity [58]. This implies that effect of HDAC6 independent of its deacetylase activity may also exist. We have given HDAC6 ZnF UBP to neuro2a cells to observe the effect on microtubule network. HDAC6 ZnF UBP treatment to neuro2a cells increased tubulin localization around nucleus in microtubule-organizing center (MTOC) (Fig. 7D). When neuro2a cells were mapped for actin and tubulin, untreated cells showed more axonal localization 
of tubulin along with actin, while HDAC6 treated cells showed tubulin predominantly in MTOC (Supp. Fig. $2 \mathrm{~A}, \mathrm{~B})$. The re-orientation of MTOC is a complex process which occurs in a dynein, cdc42 and dynactin dependent manner $[59,60]$. However, the mechanism and function of MTOC re-orientation is poorly understood. The results suggest the possible role of HDAC6 ZnF UBP domain in microtubule organization.

\section{Discussion}

Histone deactylases are known to be the enzymes that act on histones and regulates the epigenetic function in nucleus. However, many non-histone substrates of HDACs in the cytoplasm have been established [23]. Among the classes of histones deacetylase, class Ilb deacetylase HDAC6 holds a distinctive position being the major cytoplasmic deacetylase having two active catalytic domains $[23,61]$. HDAC6 exhibit domainwise function, which may or may not be dependent on each other. HDAC6 plays a major role in cell proliferation, cell migration, misfolded protein degradation and stress response through its deacetylase function or interacting with various other proteins [62]. It is a well-known fact that polyubiquitinated protein aggregates are recruited through HDAC6 ZnF UBP to be sequestered into aggresomes. HDAC6 ZnF UBP binds to diglycine motif present on ubiquitin C-terminal, which gets exposed by deubiquitinase Ataxin3 [63]. The poly-ubiquitinated aggregates are carried via dynein motor to MTOC in order to form aggresomes. The sequestered aggregates in the form of aggresomes are cleared up by autophagy pathway $[64,65]$. HDAC6 levels are found to be elevated in the AD patients following increase in the aggregate burden in cells [29]. This suggests the possibility of involvement of this domain in tackling aggregates in a deacetylase independent manner. Neuro2a cells treated with various concentrations of HDAC6 ZnF UBP to assess their viability upon treatment using MTT assay and LDH assay. Neuro2a showed no toxicity in the presence of HDAC6 ZnF UBP. pT181 and AT8 (pS202/pT205) are two pathological phosphorylation events of Tau implicated in AD [30]. Okadaic acid treatment increases the level of these two epitopes by inhibition of PP2a activity. Treatment with HDAC6 ZnF UBP decreased the levels of both pT181 and AT8. The effect possibly arises from replenishing the PP2a activity upon inhibition by OA rather than inhibition of phosphorylation [41] Class II HDACs HDAC1, HDAC6 and HDAC10 can form molecular complexes with phosphatases (PP1 or PP2a). However, HDAC6 was shown to interact only with the catalytic subunit of PP1 to form a complex retaining both phosphatase and deacetylase catalytic activity [66]. The binding of HDAC6 to PP1 was mapped to the second catalytic domain and C-terminal domain of HDAC6, which corresponds to ZnF UBP domain [41]. GSK-3 $\beta$ is a versatile protein kinase with more than hundred substrates [43]. GSK-3 $\beta$ acts on Tau in two different manners i.e. on pre-phosphorylated primed Tau and unprimed Tau [12]. In order for GSK-3 $\beta$ to work on primed substrate, the $\mathrm{N}$-terminal primed substrate binding domain provides binding-site for primed substrates up regulating the kinase activity of GSK-3ß. Phosphorylation at Serine 9 is the regulatory mechanism to down-regulate GSK-3 $\beta$ activity [11]. N-terminal domain with pSer9 acts as a pseudosubstrate for the primed substrate binding-domain of GSK-3 $\beta$ restricting the binding of other primed substrates like Tau [67]. HDAC6 ZnF UBP was found to increase the level of pSer9 
phosphorylation on GSK-3 3 indicative of down-regulated GSK-3 $\beta$ activity with HDAC6 treatment in neuro2a cells.

Cellular functions like cell growth, morphogenesis, migration, intracellular transport and attachment require co-ordinated operation of cytoskeletal networks of actin and microtubules. Both Tau and HDAC6 are known to be involved in the regulation of both these cytoskeletal networks [68]. Although HDAC6 exerts its regulatory function through catalytic domain, but the role of HDAC6 ZnF UBP domain in this aspect need to be explored. We studied the effect of HDAC6 ZnF UBP, on cytoskeletal organization and found the ability of this domain to restructure cytoskeletal network. HDAC6 ZnF UBP treatment resulted in enhancement of neuritic extensions. HDAC6 is an important modulator of cell migration and structure by associating with actin and tubulin polymerization machinery of cells $[24,58]$. We observed the colocalization of actin and HDAC6 in the neurite extensions and formation of podosomes structures involved in cell migration in HDAC6 ZnF UBP treated cells. Podosomes are membranes invaginations formed in cells like macrophages, dendritic, smooth muscle, invasive cancer cells and in post-synaptic apparatus [69] The results showed the ability of HDAC6 ZnF UBP to enhance cell migration and neurite extensions by promoting the actin assembly along cell periphery. The role of HDAC6 in cytoskeletal organization has been studied extensively. HDAC6 has been reported to play regulatory role in chemotactic movement and migration of lymphocytes in deacetylase independent manner [70]. HDAC6 also regulate the membrane ruffle formation in HSP90 and Rac1 mediated mechanism and found to be localized in membrane ruffles along with actin [71]. In this aspect, the role of HDAC6 ZnF UBP in promoting neuritic extensions by actin remodelling can prove to be a novel aspect of HDAC6 in neuroprotection. HDAC6 ZnF UBP treatment also leads to enhanced localization of tubulin around nucleus signifying the possible structural and pathological aspect of HDAC6 ZnF UBP. The function of HDAC6 ZnF UBP domain is known with respect to aggregate clearance by mediating aggresome formation. However, current findings suggest that the domain may have other regulatory functions (Fig. 8). Overall, our studies suggest the role of HDAC6 ZnF UBP domain in modulating Tau protein phosphorylation and its cytoskeletal organization which are independent of HDAC6 catalytic activity.

\section{Conclusion}

We studied the role of HDAC6 ZnF UBP domain in different aspects of AD pathology. Upon HDAC6 ZnF UBP treatment, inactive phosphorylated form of GSK-3 $\beta$ increases without any change in total GSK-3 $\beta$ level. Decreased level of Tau phospho-epitopes pT181 and AT8 with HDAC6 ZnF UBP treatment indicates that it has a regulatory role in Tau phosphorylation. Also, HDAC6 ZnF UBP was found to be involved in cytoskeletal re-organization by modulating actin dynamics and tubulin localization. Enhanced neuritic extension and formation of podosome-like structure were observed with HDAC6 ZnF UBP treatment and tubulin was more localized in MTOC suggesting its regulatory function. ApoE translocation in nucleus enhanced upon HDAC6 ZnF UBP treatment implying its possible role in ApoE pathological cascade in AD. Overall, our study suggests that the $\mathrm{ZnF}$ domain of HDAC6 performs various regulatory functions apart from its classical function in aggresome formation in protein misfolding diseases. 


\section{Declarations}

\section{Ethics approval and consent to participate}

Not applicable

\section{Consent for publication}

Not applicable

\section{Availability of data and materials}

All data generated or analyzed during this study are included in this manuscript and its supplementary information files.

\section{Competing interests}

The authors declare that they have no competing interests.

\section{Funding}

This project is supported in part by grants from the Department of Biotechnology from Neuroscience Task Force (Medical Biotechnology-Human Development \& Disease Biology (DBT-HDDB))BT/PR/19562/MED/122/13/2016 and in-house CSIR-National Chemical Laboratory grant MLP029526.

\section{Acknowledgments}

Abhishek Ankur Balmik acknowledges the Shyama Prasad Mukherjee fellowship (SPMF) from Council of Scientific Industrial Research (CSIR), India. Shweta Kishor Sonawane acknowledges Department of Biotechnology for fellowship. Tau constructs were kindly gifted by Prof. Roland Brandt from University of Osnabruck, Germany. HDAC6 ZnF UBP construct was kindly gifted by Prof. Cheryl Aerosmith, University of Toronto, Canada.

\section{Author contributions}

$\mathrm{AB}, \mathrm{SS}$ and SC conducted most of the experiments, analyzed the results, and wrote the paper. SC designed, conceived, resource provided and supervised the project and wrote the paper. All authors read and approved the final manuscript.

\section{AUTHOR INFORMATION}

\section{Author details}

\section{Abhishek Ankur Balmik}


Neurobiology Group, Division of Biochemical Sciences, CSIR-National Chemical Laboratory, Dr. Homi Bhabha Road, 411008 Pune, India

Academy of Scientific and Innovative Research (AcSIR), 411008 Pune, India

\section{Shweta Kishor Sonawane}

Neurobiology Group, Division of Biochemical Sciences, CSIR-National Chemical Laboratory, Dr. Homi Bhabha Road, 411008 Pune, India

Academy of Scientific and Innovative Research (AcSIR), 411008 Pune, India

Subashchandrabose Chinnathambi

Neurobiology Group, Division of Biochemical Sciences, CSIR-National Chemical Laboratory, Dr. Homi Bhabha Road, 411008 Pune, India

Academy of Scientific and Innovative Research (AcSIR), 411008 Pune, India

Corresponding Author

Correspondence and requests for materials should be addressed to Prof. Subashchandrabose Chinnathambi. Email: s.chinnathambi@ncl.res.in Telephone: +91-20-25902232, Fax.+91-20-25902648.

ORCID

Subashchandrabose Chinnathambi: 0000-0002-5468-2129

\section{References}

1. Williams D: Tauopathies: classification and clinical update on neurodegenerative diseases associated with microtubule-associated protein tau. Internal medicine journal 2006, 36:652-660.

2. Coppola G, Chinnathambi S, Lee JJ, Dombroski BA, Baker MC, Soto-Ortolaza Al, Lee SE, Klein E, Huang AY, Sears R: Evidence for a role of the rare p. A152T variant in MAPT in increasing the risk for FTD-spectrum and Alzheimer's diseases. Human molecular genetics 2012, 21:3500-3512.

3. Ksiezak-Reding $\mathrm{H}$, Yen S-H: Structural stability of paired helical filaments requires microtubulebinding domains of tau: a model for self-association. Neuron 1991, 6:717-728.

4. Iqbal K, Liu F, Gong C-X, Grundke-lqbal I: Tau in Alzheimer disease and related tauopathies. Current Alzheimer Research 2010, 7:656-664.

5. Goedert M, Ghetti B, Spillantini MG: Frontotemporal dementia: implications for understanding Alzheimer disease. Cold Spring Harbor perspectives in medicine 2012, 2:a006254.

6. Martin L, Latypova X, Terro F: Post-translational modifications of tau protein: implications for Alzheimer's disease. Neurochemistry international 2011, 58:458-471. 
7. Biernat J, Gustke N, Drewes G, Mandelkow E: Phosphorylation of Ser262 strongly reduces binding of tau to microtubules: distinction between PHF-like immunoreactivity and microtubule binding. Neuron 1993, 11:153-163.

8. Biernat J, Mandelkow E, Schröter C, Lichtenberg-Kraag B, Steiner B, Berling B, Meyer H, Mercken M, Vandermeeren $A$, Goedert M: The switch of tau protein to an Alzheimer-like state includes the phosphorylation of two serine-proline motifs upstream of the microtubule binding region. The EMBO journal 1992, 11:1593-1597.

9. Drewes G, Ebneth A, Preuss U, Mandelkow E-M, Mandelkow E: MARK, a novel family of protein kinases that phosphorylate microtubule-associated proteins and trigger microtubule disruption. Cell 1997, 89:297-308.

10. Lucas JJ, Hernández F, Gómez-Ramos P, Morán MA, Hen R, Avila J: Decreased nuclear $\beta$-catenin, tau hyperphosphorylation and neurodegeneration in GSK-3 $\beta$ conditional transgenic mice. The EMBO journal 2001, 20:27-39.

11. Cho JH, Johnson GV: Primed phosphorylation of tau at Thr231 by glycogen synthase kinase $3 \beta$ (GSK3 $\beta$ ) plays a critical role in regulating tau's ability to bind and stabilize microtubules. Journal of neurochemistry 2004, 88:349-358.

12. Cho J-H, Johnson GV: Glycogen Synthase Kinase $3 \beta$ Phosphorylates tau at both primed and unprimed sites differential impact on microtubule binding. Journal of Biological Chemistry 2003, 278:187-193.

13. Mandelkow E-M, Mandelkow E: Biochemistry and cell biology of tau protein in neurofibrillary degeneration. Cold Spring Harbor perspectives in medicine 2012, 2:a006247.

14. Amm I, Sommer T, Wolf DH: Protein quality control and elimination of protein waste: the role of the ubiquitin-proteasome system. Biochimica et Biophysica Acta (BBA)-Molecular Cell Research 2014, 1843:182-196.

15. Pierre S-R, Vernace V, Wang Z, Figueiredo-Pereira ME: Assembly of protein aggregates in neurodegeneration: mechanisms linking the ubiquitin/proteasome pathway and chaperones. In Madame Curie Bioscience Database [Internet]. Landes Bioscience; 2013

16. Ciechanover A, Kwon YT: Degradation of misfolded proteins in neurodegenerative diseases: therapeutic targets and strategies. Experimental \& molecular medicine 2015, 47:e147.

17. Gorantla NV, Chinnathambi S: Tau Protein Squired by Molecular Chaperones During Alzheimer's Disease. Journal of Molecular Neuroscience 2018, 66:356-368.

18. Tai H-C, Schuman EM: Ubiquitin, the proteasome and protein degradation in neuronal function and dysfunction. Nature Reviews Neuroscience 2008, 9:826.

19. Casadei N, Pöhler A-M, Tomás-Zapico C, Torres-Peraza J, Schwedhelm I, Witz A, Zamolo I, De Heer R, Spruijt B, Noldus LP: Overexpression of synphilin-1 promotes clearance of soluble and misfolded alpha-synuclein without restoring the motor phenotype in aged $\mathrm{A} 30 \mathrm{P}$ transgenic mice. Human molecular genetics 2013, 23:767-781. 
20. Ardley HC, Scott GB, Rose SA, Tan NG, Robinson PA: UCH-L1 aggresome formation in response to proteasome impairment indicates a role in inclusion formation in Parkinson's disease. Journal of neurochemistry 2004, 90:379-391.

21. Simões-Pires C, Zwick V, Nurisso A, Schenker E, Carrupt P-A, Cuendet M: HDAC6 as a target for neurodegenerative diseases: what makes it different from the other HDACs? Molecular neurodegeneration 2013, 8:7.

22. Verdin E, Dequiedt F, Kasler HG: Class II histone deacetylases: versatile regulators. TRENDS in Genetics 2003, 19:286-293.

23. Yao Y-L, Yang W-M: Beyond histone and deacetylase: an overview of cytoplasmic histone deacetylases and their nonhistone substrates. BioMed Research International 2010, 2011.

24. Zhang X, Yuan Z, Zhang Y, Yong S, Salas-Burgos A, Koomen J, Olashaw N, Parsons JT, Yang X-J, Dent SR: HDAC6 modulates cell motility by altering the acetylation level of cortactin. Molecular cell 2007, 27:197-213.

25. Pandey UB, Batlevi Y, Baehrecke EH, Taylor JP: HDAC6 at the intersection of autophagy, the ubiquitinproteasome system, and neurodegeneration. Autophagy 2007, 3:643-645.

26. Pandey UB, Nie Z, Batlevi Y, McCray BA, Ritson GP, Nedelsky NB, Schwartz SL, DiProspero NA, Knight MA, Schuldiner O: HDAC6 rescues neurodegeneration and provides an essential link between autophagy and the UPS. Nature 2007, 447:860.

27. Hao R, Nanduri P, Rao Y, Panichelli RS, Ito A, Yoshida M, Yao T-P: Proteasomes activate aggresome disassembly and clearance by producing unanchored ubiquitin chains. Molecular cell 2013, 51:819828.

28. Iwata A, Riley BE, Johnston JA, Kopito RR: HDAC6 and microtubules are required for autophagic degradation of aggregated huntingtin. Journal of Biological Chemistry 2005, 280:40282-40292.

29. Zhang L, Sheng S, Qin C: The role of HDAC6 in Alzheimer's disease. Journal of Alzheimer's Disease 2013, 33:283-295.

30. Šimić G, Babić Leko M, Wray S, Harrington C, Delalle I, Jovanov-Milošević N, Bažadona D, Buée L, De Silva R, Di Giovanni G: Tau protein hyperphosphorylation and aggregation in Alzheimer's disease and other tauopathies, and possible neuroprotective strategies. Biomolecules 2016, 6:6.

31. Šimić G, Diana A, Hof PR: Phosphorylation pattern of tau associated with distinct changes of the growth cone cytoskeleton. In Guidance Cues in the Developing Brain. Springer; 2003: 33-48

32. Arendt T, Holzer M, Fruth R, Brückner M, Gärtner U: Paired helical filament-like phosphorylation of tau, deposition of $\beta / A 4$-amyloid and memory impairment in rat induced by chronic inhibition of phosphatase 1 and 2A. Neuroscience 1995, 69:691-698.

33. Sontag E, Hladik C, Montgomery L, Luangpirom A, Mudrak I, Ogris E, White III CL: Downregulation of protein phosphatase 2A carboxyl methylation and methyltransferase may contribute to Alzheimer disease pathogenesis. Journal of Neuropathology \& Experimental Neurology 2004, 63:1080-1091.

34. Vintém APB, Henriques AG, e Silva OAdC, e Silva EFdC: PP1 inhibition by A $\beta$ peptide as a potential pathological mechanism in Alzheimer's disease. Neurotoxicology and teratology 2009, 31:85-88. 
35. Li B, Chohan MO, Grundke-lqbal I, Iqbal K: Disruption of microtubule network by Alzheimer abnormally hyperphosphorylated tau. Acta neuropathologica 2007, 113:501-511.

36. Penzes P, VanLeeuwen J-E: Impaired regulation of synaptic actin cytoskeleton in Alzheimer's disease. Brain research reviews 2011, 67:184-192.

37. Fiala JC, Spacek J, Harris KM: Dendritic spine pathology: cause or consequence of neurological disorders? Brain research reviews 2002, 39:29-54.

38. Selkoe DJ: Alzheimer's disease is a synaptic failure. Science 2002, 298:789-791.

39. Boyault C, Sadoul K, Pabion M, Khochbin S: HDAC6, at the crossroads between cytoskeleton and cell signaling by acetylation and ubiquitination. Oncogene 2007, 26:5468.

40. Alao JP, Stavropoulou AV, Lam EW, Coombes RC: Role of glycogen synthase kinase 3 beta (GSK3 $\beta$ ) in mediating the cytotoxic effects of the histone deacetylase inhibitor trichostatin A (TSA) in MCF-7 breast cancer cells. Molecular cancer 2006, 5:40.

41. Brush MH, Guardiola A, Connor JH, Yao T-P, Shenolikar S: Deactylase inhibitors disrupt cellular complexes containing protein phosphatases and deacetylases. Journal of Biological Chemistry 2004, 279:7685-7691.

42. Beurel E: HDAC6 regulates LPS-tolerance in astrocytes. PLoS One 2011, 6:e25804.

43. Beurel E, Grieco SF, Jope RS: Glycogen synthase kinase-3 (GSK3): regulation, actions, and diseases. Pharmacology \& therapeutics 2015, 148:114-131.

44. Avila J: Tau kinases and phosphatases. Journal of cellular and molecular medicine 2008, 12:258259.

45. Boban M, Leko MB, Miškić T, Hof PR, Šimić G: Human neuroblastoma SH-SY5Y cells treated with okadaic acid express phosphorylated high molecular weight tau-immunoreactive protein species. Journal of neuroscience methods 2018.

46. Oda T, Iwasa M, Aihara T, Maéda Y, Narita A: The nature of the globular-to fibrous-actin transition. Nature 2009, 457:441.

47. Lee SH, Dominguez R: Regulation of actin cytoskeleton dynamics in cells. Molecules and cells 2010, 29:311-325.

48. Moghaddam HS, Aarabi MH: Aß-Mediated Dysregulation of F-Actin Nanoarchitecture Leads to Loss of Dendritic Spines and Alzheimer's Disease-Related Cognitive Impairments. Journal of Neuroscience 2018, 38:5840-5842.

49. Biswas S, Kalil K: The microtubule-associated protein tau mediates the organization of microtubules and their dynamic exploration of actin-rich lamellipodia and filopodia of cortical growth cones. Journal of Neuroscience 2018, 38:291-307.

50. Gimona M, Buccione R, Courtneidge SA, Linder S: Assembly and biological role of podosomes and invadopodia. Current opinion in cell biology 2008, 20:235-241.

51. Siddiqui TA, Lively S, Vincent C, Schlichter LC: Regulation of podosome formation, microglial

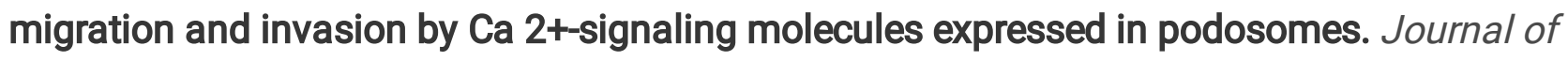


neuroinflammation 2012, 9:250.

52. Sen A, Nelson TJ, Alkon DL: ApoE4 and A $\beta$ oligomers reduce BDNF expression via HDAC nuclear translocation. Journal of Neuroscience 2015, 35:7538-7551.

53. Jiang Q, Lee CD, Mandrekar S, Wilkinson B, Cramer P, Zelcer N, Mann K, Lamb B, Willson TM, Collins $\mathrm{JL}$ : ApoE promotes the proteolytic degradation of AB. Neuron 2008, 58:681-693.

54. Kim WS, Elliott DA, Kockx M, Kritharides L, Rye K-A, Jans DA, Garner B: Analysis of apolipoprotein E nuclear localization using green fluorescent protein and biotinylation approaches. Biochemical Journal 2008, 409:701-709.

55. Rohn TT, Moore ZD: Nuclear localization of apolipoprotein E4: a new trick for an old protein. International journal of neurology and neurotherapy 2017, 4.

56. Semenkovich CF, Ostlund Jr RE, Olson MO, Yang JW: A protein partially expressed on the surface of HepG2 cells that binds lipoproteins specifically is nucleolin. Biochemistry 1990, 29:9708-9713.

57. Wang S-A, Li H-Y, Hsu T-I, Chen S-H, Wu C-J, Chang W-C, Hung J-J: Heat shock protein 90 stabilizes nucleolin to increase mRNA stability in mitosis. Journal of Biological Chemistry 2011, 286:4381643829.

58. Zilberman Y, Ballestrem C, Carramusa L, Mazitschek R, Khochbin S, Bershadsky A: Regulation of microtubule dynamics by inhibition of the tubulin deacetylase HDAC6. Journal of cell science 2009, 122:3531-3541.

59. Sancho D, Vicente-Manzanares M, Mittelbrunn M, Montoya MC, Gordón-Alonso M, Serrador JM, Sánchez-Madrid F: Regulation of microtubule-organizing center orientation and actomyosin cytoskeleton rearrangement during immune interactions. Immunological reviews 2002, 189:84-97.

60. Palazzo AF, Joseph HL, Chen Y-J, Dujardin DL, Alberts AS, Pfister KK, Vallee RB, Gundersen GG: Cdc42, dynein, and dynactin regulate MTOC reorientation independent of Rho-regulated microtubule stabilization. Current Biology 2001, 11:1536-1541.

61. Li M, Zhuang Y, Shan B: Analysis of expression and functions of histone deacetylase 6 (hdac6). In Histone Deacetylases. Springer; 2016: 85-94

62. Li Y, Shin D, Kwon SH: Histone deacetylase 6 plays a role as a distinct regulator of diverse cellular processes. The FEBS journal 2013, 280:775-793.

63. Ouyang H, Ali YO, Ravichandran M, Dong A, Qiu W, MacKenzie F, Dhe-Paganon S, Arrowsmith $\mathrm{CH}$, Zhai RG: Protein aggregates are recruited to aggresome by histone deacetylase 6 via unanchored ubiquitin C termini. Journal of Biological Chemistry 2012, 287:2317-2327.

64. Yan J: Interplay between HDAC6 and its interacting partners: essential roles in the aggresomeautophagy pathway and neurodegenerative diseases. DNA and cell biology 2014, 33:567-580.

65. Van Helleputte L, Benoy V, Van Den Bosch L: The role of histone deacetylase 6 (HDAC6) in neurodegeneration. Res Rep Biol 2014, 5:1-13.

66. Esteves SL, Domingues SC, da Cruz e Silva OA, Fardilha M, da Cruz e Silva EF: Protein phosphatase 1a interacting proteins in the human brain. Omics: a journal of integrative biology 2012, 16:3-17. 
67. Fang X, Yu SX, Lu Y, Bast RC, Woodgett JR, Mills GB: Phosphorylation and inactivation of glycogen synthase kinase 3 by protein kinase A. Proceedings of the National Academy of Sciences 2000, 97:11960-11965.

68. Elie A, Prezel E, Guérin C, Denarier E, Ramirez-Rios S, Serre L, Andrieux A, Fourest-Lieuvin A, Blanchoin L, Arnal I: Tau co-organizes dynamic microtubule and actin networks. Scientific reports 2015, 5:9964.

69. Vincent C, Siddiqui TA, Schlichter LC: Podosomes in migrating microglia: components and matrix degradation. Journal of neuroinflammation 2012, 9:190.

70. Cabrero JR, Serrador JM, Barreiro O, Mittelbrunn M, Naranjo-Suárez S, Martín-Cófreces N, VicenteManzanares M, Mazitschek R, Bradner JE, Ávila J: Lymphocyte chemotaxis is regulated by histone deacetylase 6 , independently of its deacetylase activity. Molecular biology of the cell 2006, 17:34353445 .

71. Gao Y-s, Hubbert CC, Lu J, Lee Y-S, Lee J-Y, Yao T-P: Histone deacetylase 6 regulates growth factorinduced actin remodeling and endocytosis. Molecular and cellular biology 2007, 27:8637-8647.

72. Gorantla NV, Khandelwal P, Poddar P, Chinnathambi S: Global conformation of tau protein mapped by Raman spectroscopy. In Tau Protein. Humana Press: Springer; 2017: 21-31

73. Gorantla NV, Shkumatov AV, Chinnathambi S: Conformational Dynamics of Intracellular Tau Protein Revealed by CD and SAXS. In Tau Protein. Springer; 2017: 3-20

\section{Supplementary Information}

Additional file 1: Supplementary figure 1. Internalization of HDAC6 ZnF UBP in neuro2a. Supplementary figure 2. Co-localization of actin and tubulin in neuro2a. Supplementary figure 3. DIC for neurite extensions in HDAC6 ZnF UBP treated cells.

\section{Figures}




\section{Figure 1}

A

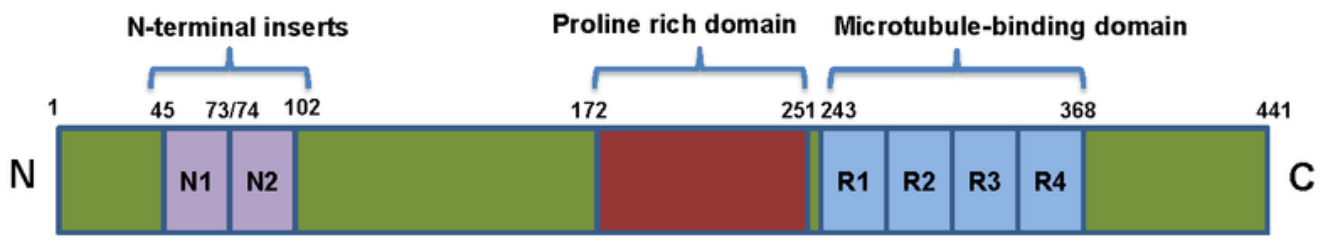

B

HDAC $6^{\mathrm{ZnF} \text { UBP }}$

MGSSHHHHHH SSGLVPRGSP LPWCPHLVAV

CPI PAAGLDV TQPCGDCGTI QENWVCLSCY QVYCGRYING HMLQHHGNSG HPLVLSYIDL SAWCYYCQAY VHHQALLDVK NIAHQNKFGE DMPHPH

D

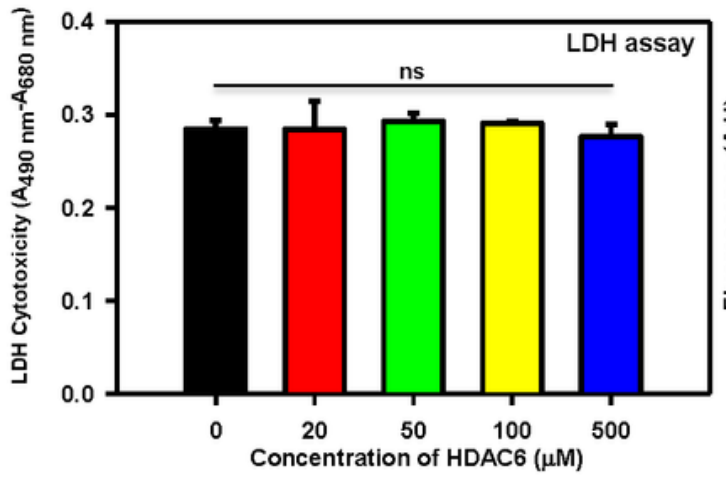

C

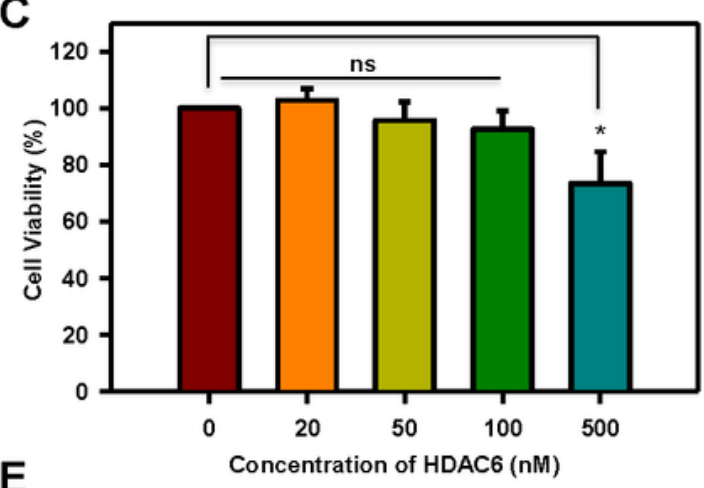

E

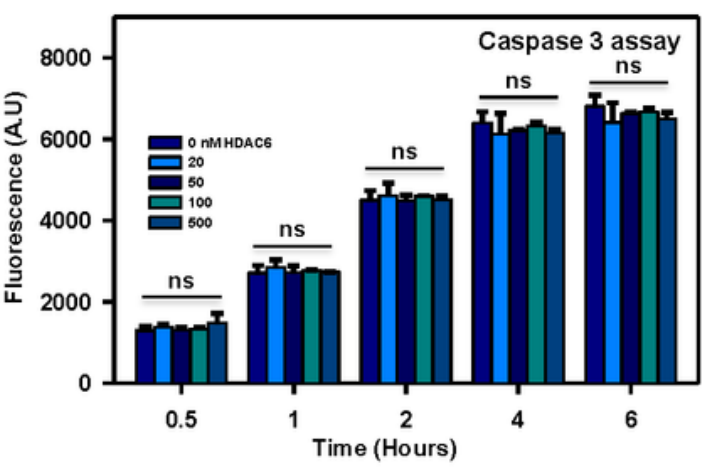

Figure 1

HDAC6 ZnF UBP treatment to neuro2a cells does not have any toxic effect. A) Bar diagram for the human microtubule-associated protein Tau comprising of two N-terminals inserts and four repeat regions preceded by a polyproline domain. B) Amino acid sequence of human HDAC6 Zinc finger ubiquitinbinding domain. This domain is located on the C-terminal of HDAC6 and associates with polyubiquitinated protein aggregates to mediate the formation of aggresomes. C) MTT assay was carried 
out to determine the viability of neuro2a upon HDAC6 ZnF UBP treatment. Neuroblastoma cells treated with HDAC6 at different concentrations show minimum toxicity and maintain viability at $80 \%$ at highest concentration of $500 \mathrm{nM}$. D) LDH release assay determines the damage to the cell membrane upon exposure to test molecule. The membrane leakage assay (LDH assay) shows that HDAC6 does not disrupt the cell membrane and affect cell viability. E) Apoptosis rate of neuro2a cells upon HDAC6 ZnF UBP treatment was assayed using Caspase- 3 assay. Caspase-3 assay shows increased levels of caspase- 3 with successive time interval but do not differ from control samples. (ns - non-significant, * indicates $\mathrm{P} \leq 0.05)$. 


\section{Figure 2}
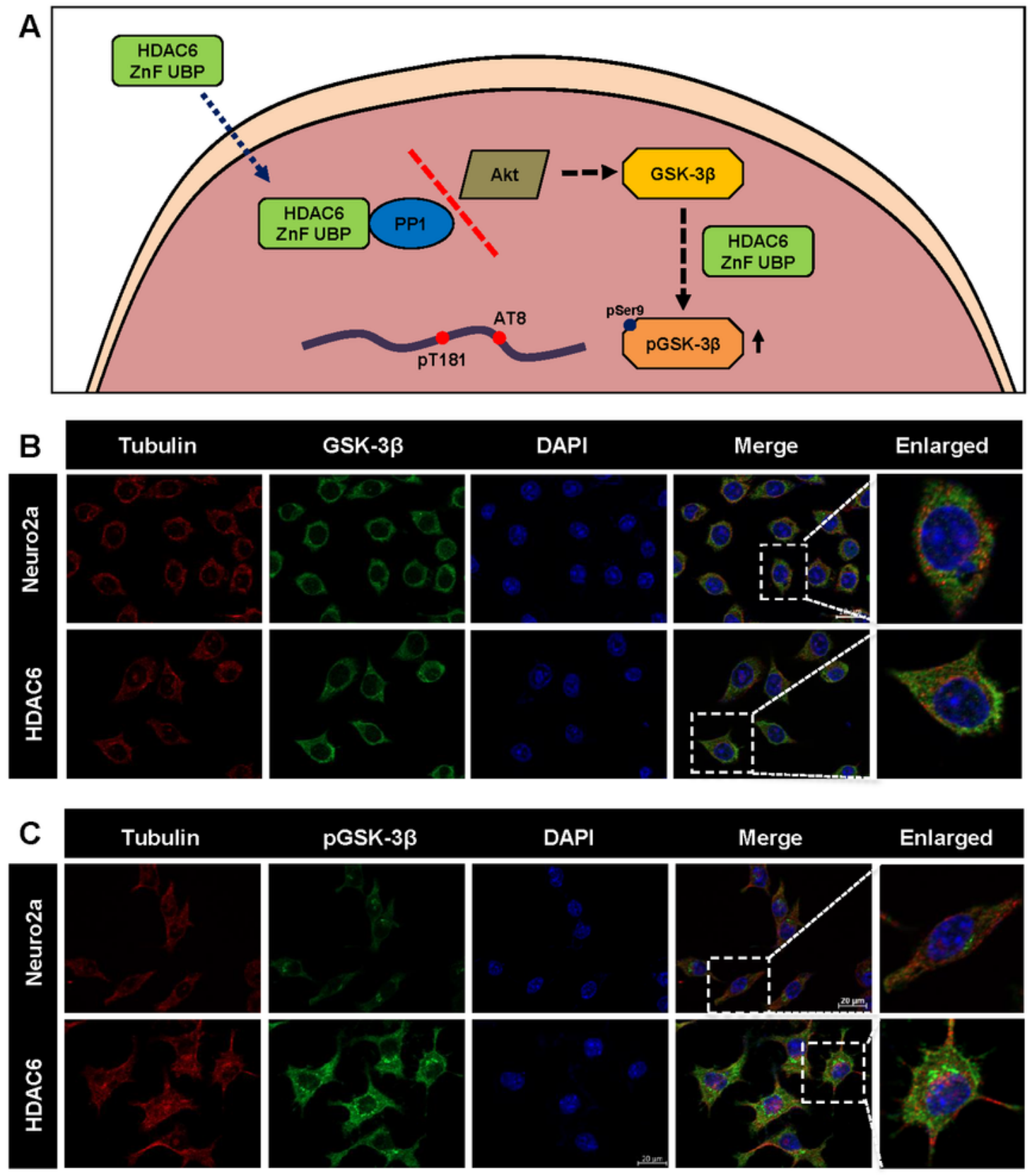

\section{Figure 2}

Downregulation of GSK-3 $\beta$ activity by HDAC6. The levels of GSK-3 $\beta$ and pGSK-3 $\beta$ reflect its activity in the cell. Enhanced levels of pGSK-3 $\beta$ were observed while total GSK-3 $\beta$ remained unaltered upon the treatment of HDAC6 ZnF UBP domain. A) Neuro2a mapped for total GSK-3 $\beta$ shows their unaltered levels upon HDAC6 ZnF UBP. B) The activity of GSK-3 $\beta$ is governed by phosphorylation at specific residues which either upregulates or downregulates its activity. Inhibitory phosphorylation of GSK-3 $\beta$ at Ser9 
increases upon HDAC6 ZnF UBP treatment. The enlarged image shows the elevated level of pGSK-3 $\beta$ compared to neuro2a cell control. C) Proposed mechanism for the effect of HDAC6 ZnF UBP on the regulation of GSK-3 $\beta$ activity. GSK-3 $\beta$ activity is negatively regulated by Akt kinase, which phosphorylates GSK-3 $\beta$ at Ser9. Akt is active in its phosphorylated state. HDAC6 ZnF UBP associates with PP1 restricting it from dephosphorylating Akt, hence resulting in its active phosphorylated state.

\section{Figure 3}
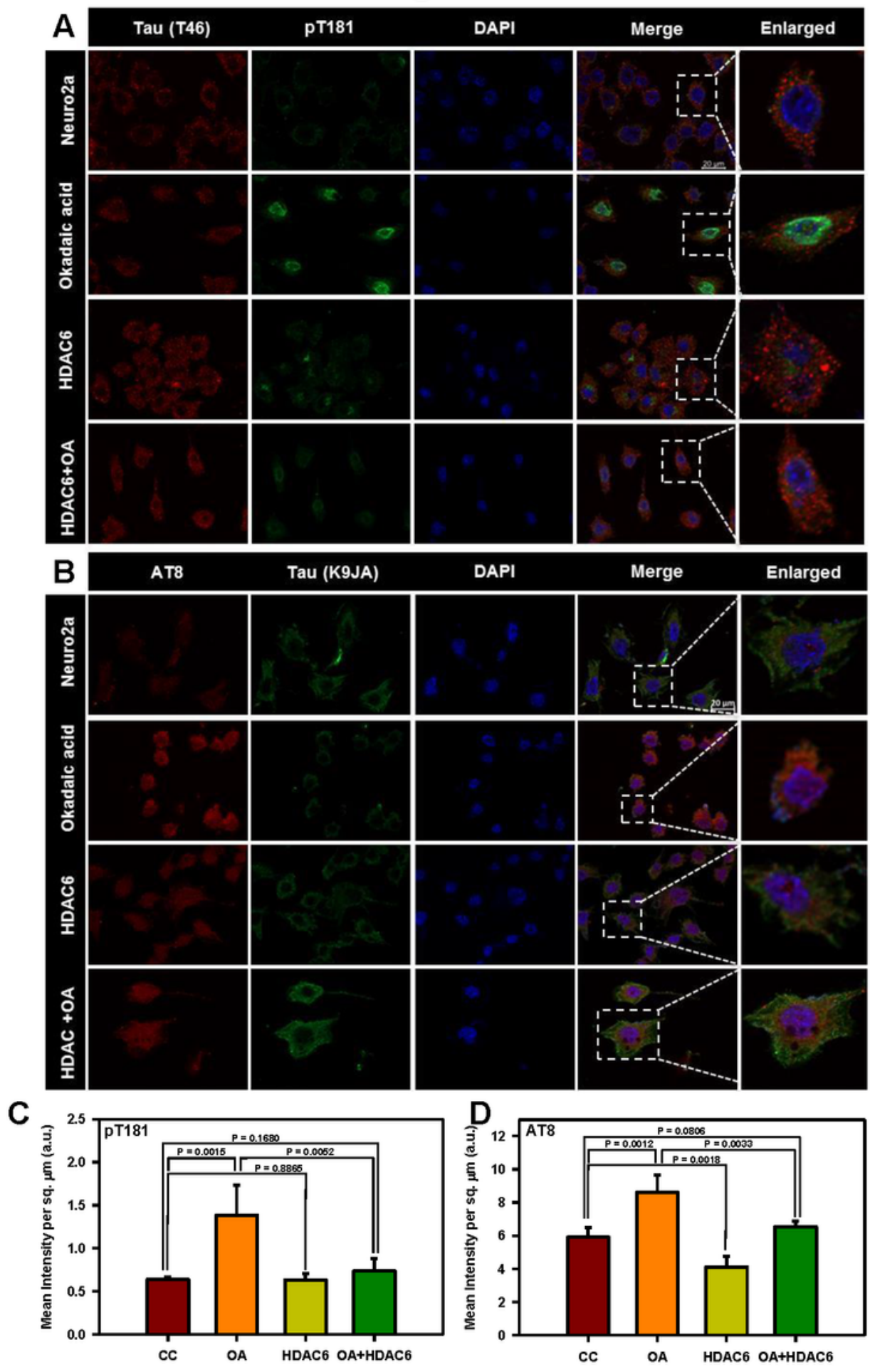

Figure 3 
Inhibition of Tau phosphorylation by HDAC6. Neuro2a cells treated with okadaic acid (OA) enhanced overall phosphorylation by inhibition of PP2A. Tau phosphorylations at pT181 and AT8 epitopes of Tau were mapped after OA treatment alone as well as in simultaneous treatment with HDAC6 ZnF UBP to observe the effect on Tau phosphorylation. A) HDAC6 treatment for 24 hours along with OA shows inhibition of Tau phosphorylation at PT181 epitope whereas OA treatment shows increased phosphorylation at PT181. B) Phosphorylation at AT8 epitope is also reduced in presence of HDAC and $O A$ as compared to $O A$ alone. The enlarged image of neuro2a treated with $O A$ alone and $O A$ along with HDAC6 ZnF UBP shows marked difference in the level of phosphorylation at pT181 and AT8 epitopes. C, D). Mean fluorescence intensity for AT8 immunostaining of untreated neuro2a (CC), okadaic acid treatment (OA), HDAC6 ZnF UBP treatment (HDAC6) and HDAc6 ZnF UBP treatment along with okadaic acid. Okadaic acid treatment resulted in increased level of pT181 and AT8 phospho-epitopes. HDAC6 ZnF UBP treatment with OA reduced the level of both phospho-epitopes. 
Figure 4
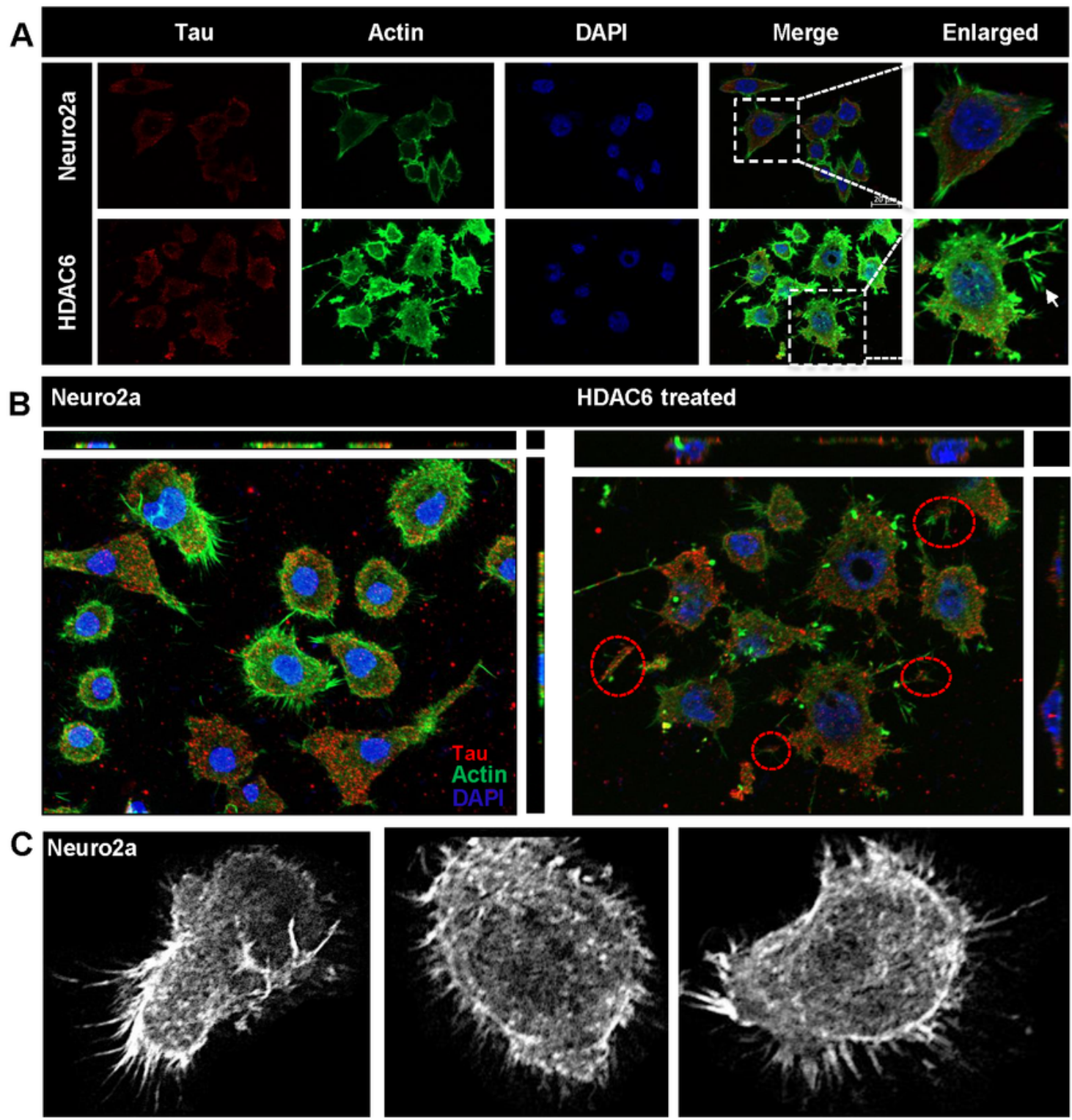

D HDAC6
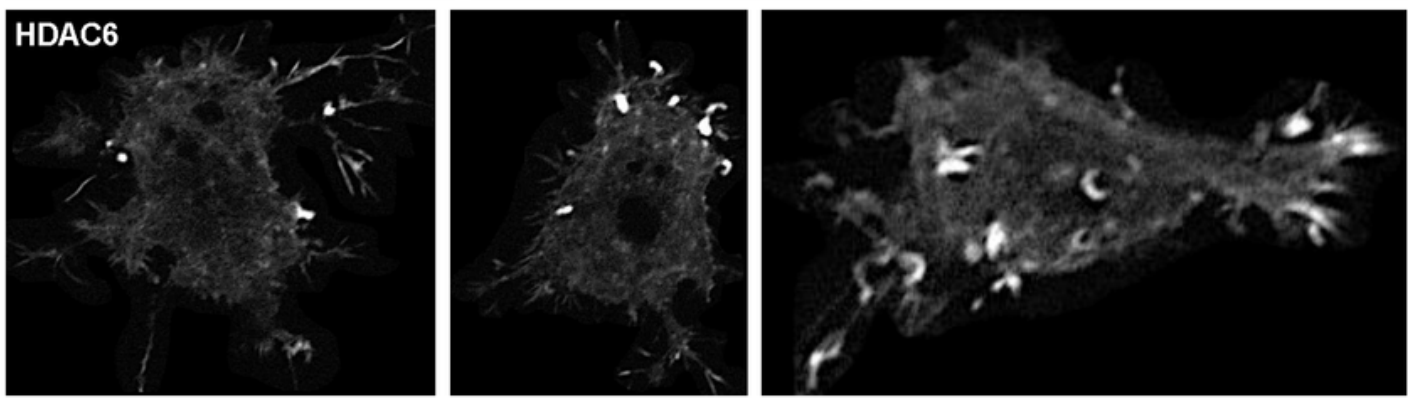

Figure 4

HDAC6 as a modulator of actin dynamics. HDAC6 deacetylase activity is known to modulate actin dynamics. The effect on actin dynamics was studied after exposure to HDAC6 ZnF UBP domain. HDAC6 ZnF UBP treatment to neuro2a resulted in enhancement of neurite extensions which are mapped by Factin staining. A) HDAC6 ZnF UBP treatment for 24 hours increases the neuronal extensions as compared to untreated control cells when the extensions are mapped by actin immunostaining. The Tau levels 
remain unaltered in both the groups. B) The orthogonal projection shows the increase in number of neurites in the HDAC6 ZnF UBP treated cells. C) Greyscale images for untreated neuro2a cells mapped for F-actin showed small extensions in the growth cones. D) In HDAC6 ZnF UBP treated cells, F-actin containing longer extensions were observed. Growth cones were observed to be concentrated in membrane extensions and filopodia-like structures.

\section{Figure 5}
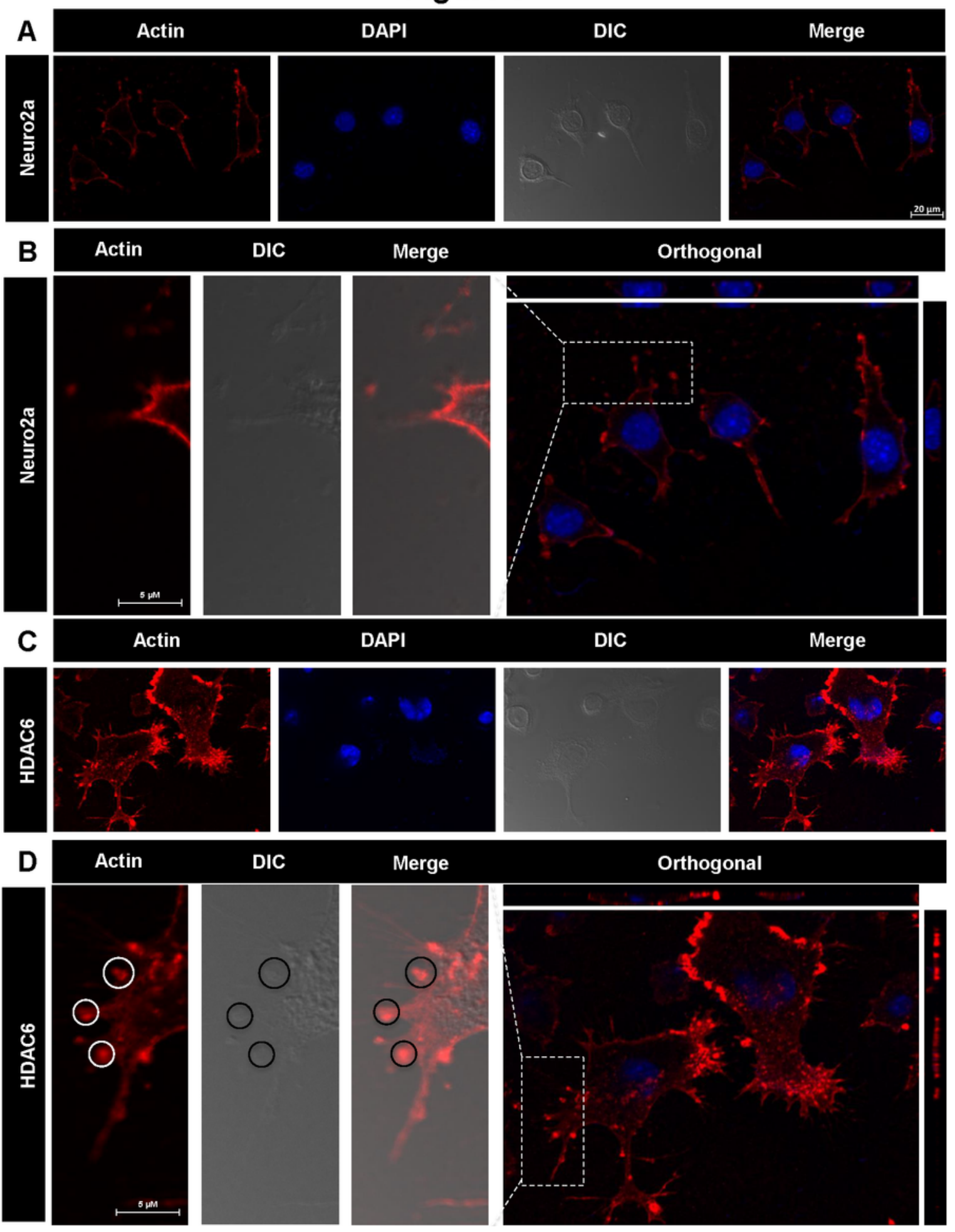

Figure 5 
Enhancement of podosome formation by HDAC6. HDAC6 deacetylase activity is known for regulating the actin dynamics in cells and podosome formation. Structures resembling podosomes and podonuts were observed in neuro2a upon HDAC6 ZnF UBP treatment. Podosomes and podonuts are actin rich structures involved in cell attachment and migration. A) Podosomes marked by the actin rich structures along plasma membrane, were observed in neuro2a cells at a minimal level. B) Enhancement in podosome like structures observed in HDAC6 ZnF UBP treated cells. Orthogonal projection images showed marked difference in the membrane morphology and actin concentrated in membrane ruffles and podosome like structures in HDAC6 ZnF UBP treated cells. C) HDAC6 is found to be present in the neuritic extensions along with actin suggesting its role in regulation of neurite extensions. This is not seen in case of untreated cells as indicated in the enlarged images for both groups. D) In HDAC6 ZnF UBP treated cells, Factin containing longer extensions were observed. Growth cones were observed to be concentrated in membrane extensions and filopodia-like structures. 


\section{Figure 6}
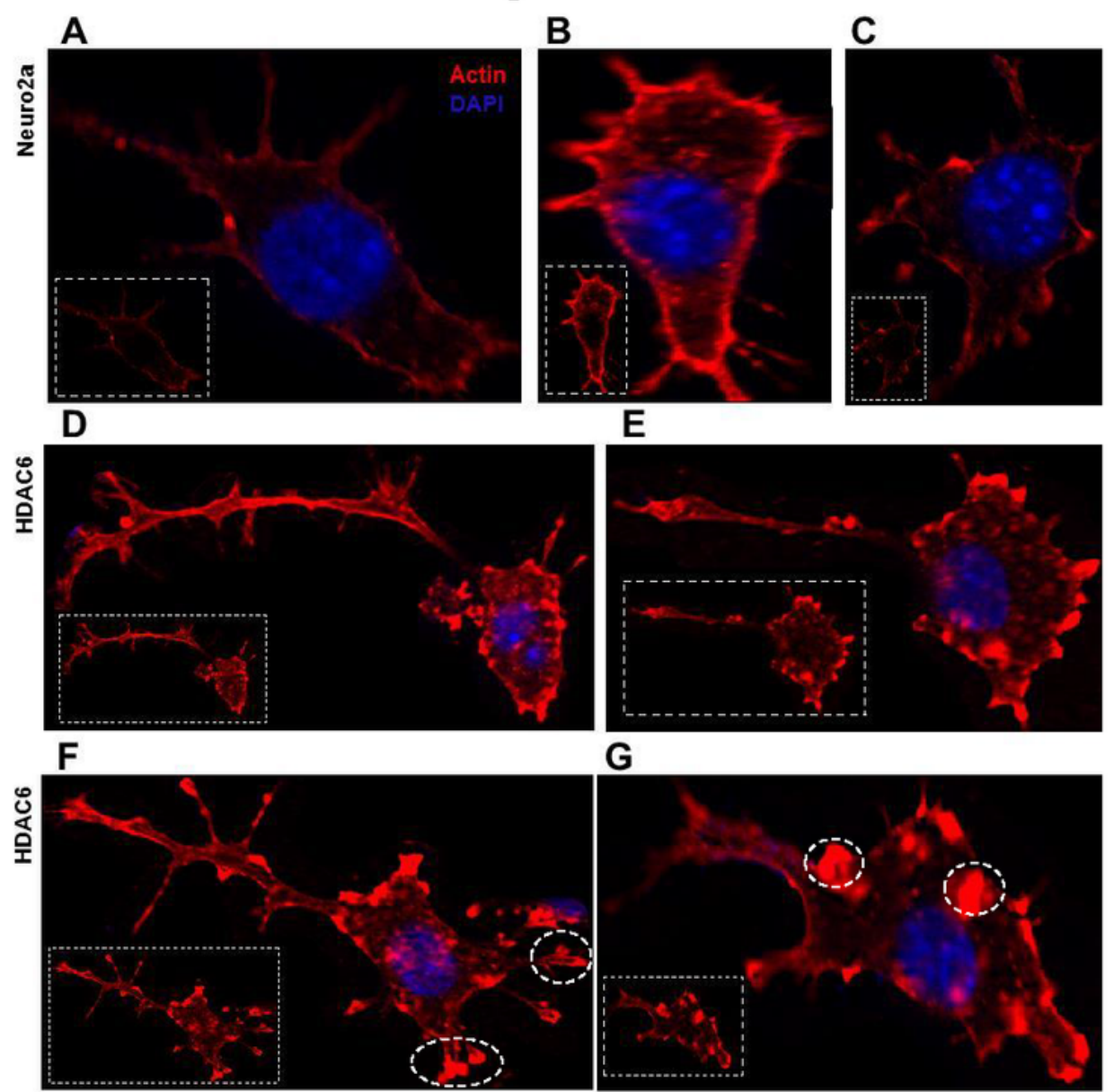

G
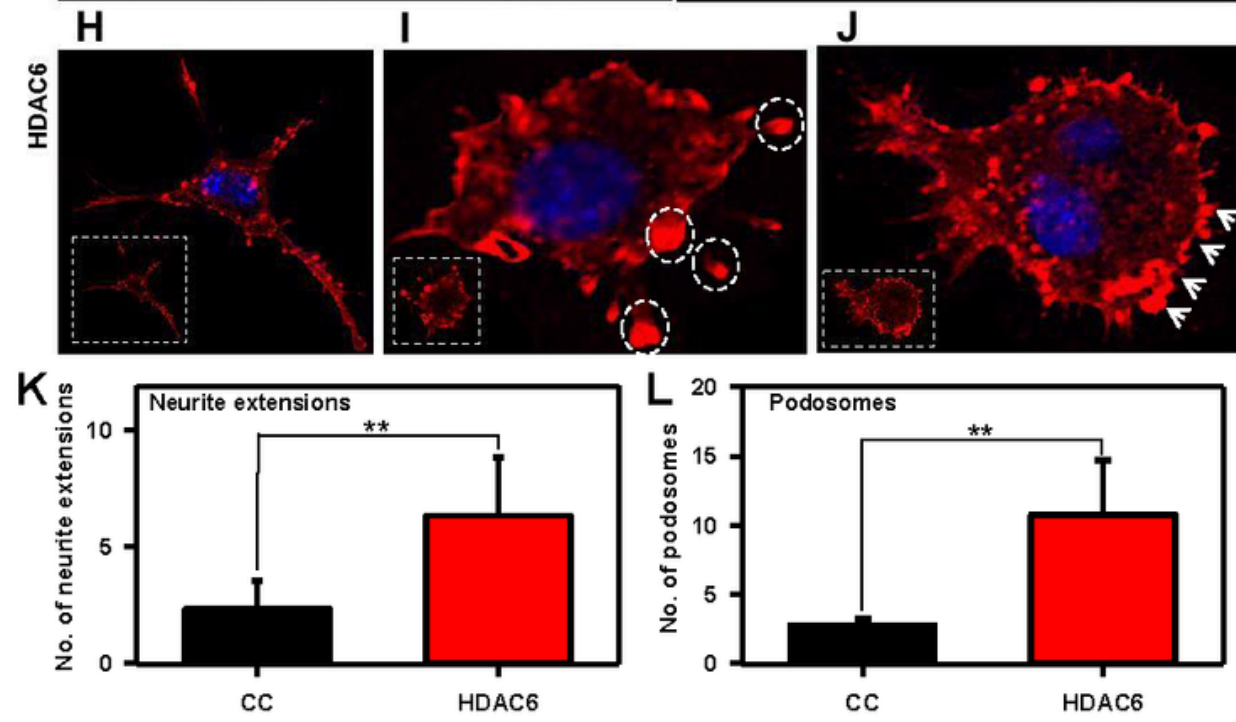

Figure 6

Podosome, lamellipodia and podonut-like structure induced by HDAC6 ZnF UBP. Neuro2a cells exposed to HDAC6 ZnF UBP exhibit a variety of actin rich structures characteristics of migratory or invading cells. A, $B, C)$ Untreated neuro2a cells showed actin uniformly distributed along the periphery with small neuritic extensions while D, E) HDAC6 ZnF UBP treated cells showed longer extensions and membranes ruffles resembling podosomes involved in cell migration. F, G) Invadopodia and lamellipodia-like structures 
(encircled) were also observed, which are found in phagocytic cells. $\mathrm{H}, \mathrm{I}, \mathrm{J}$ ) Most of the treated cells were observed to consist of assemblance of podosomes (encircled) and podosome clusters called podonuts (white arrow) rich in actin. K) The overall neurite extensions in neuro2a cells were counted in untreated and HDAC6 treated group in $\beta$-actin immunostained cells. The number of extensions in HDAC6 treated cells were found to be greatly enhanced compared to untreated group. L) The podosomes formed after HDAC6 ZnF UBP treatment were quantified by counting the podosome crown structures in both untreated and treated groups. HDAC6 ZnF UBP treated cells showed more podosome clusters signifying its actin modulating effect. 
Figure 7
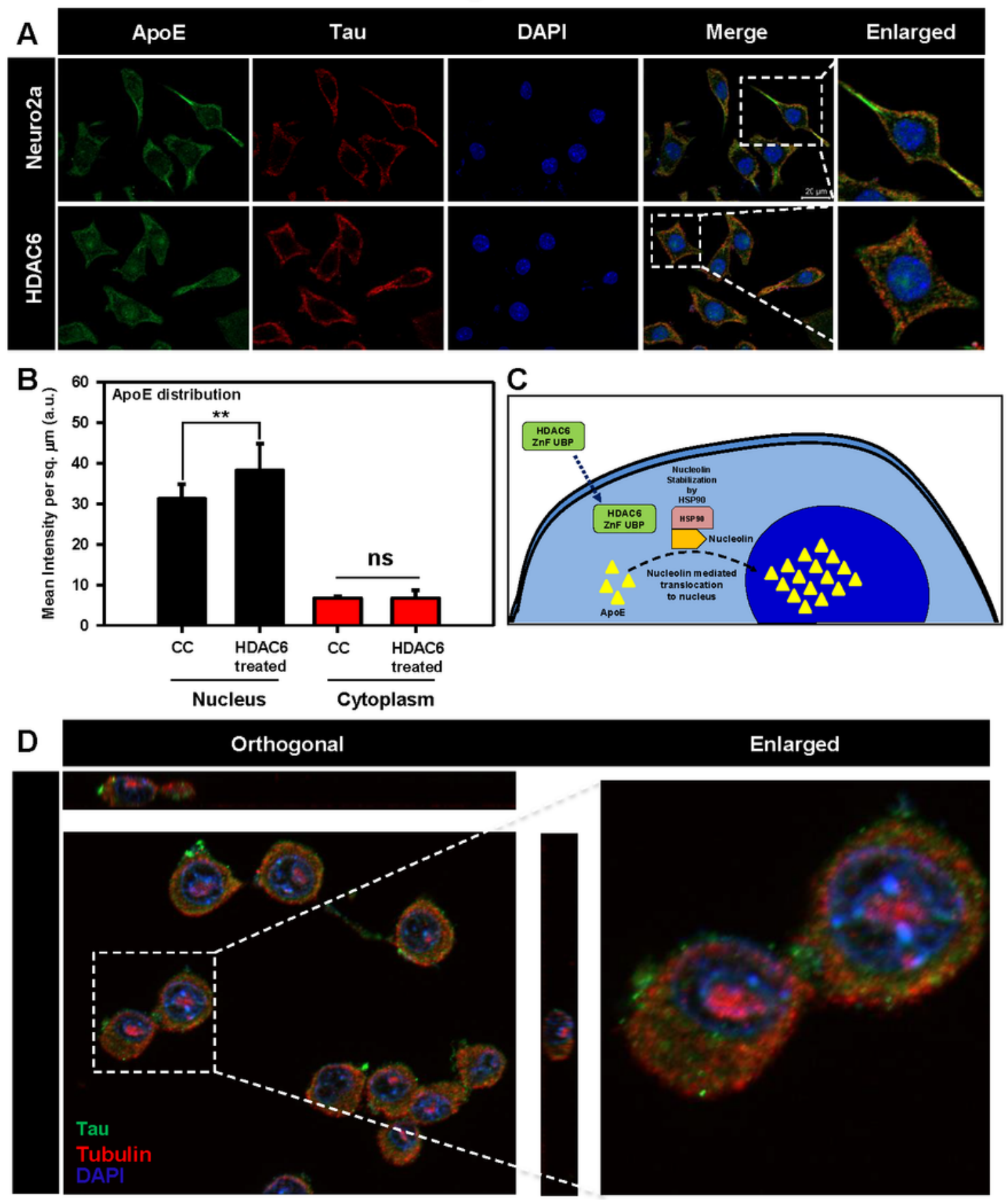

Enlarged

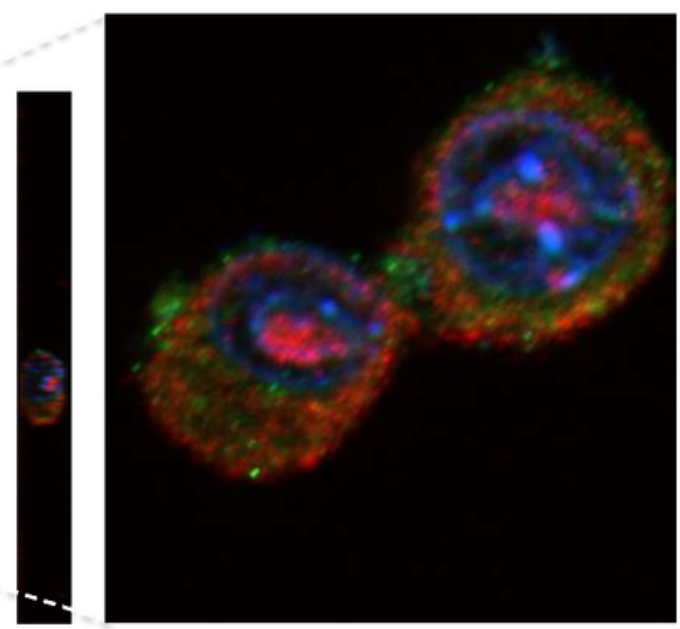

Figure 7

Modulation of ApoE and Tubulin localization by HDAC6. Cells were mapped for ApoE to observe its level and localization. Enhanced nuclear localization of ApoE was observed with HDAC6 ZnF UBP treatment. HDAC6 ZnF UBP also affected the tubulin distribution where the treated cells showed more tubulin localization in MTOC compared to untreated cells where tubulin was evenly distributed. A) Immunofluorescence mapping for ApoE and Tau upon HDAC6 treatment in neuro2a cells shows 
increased nuclear localization of ApoE. B) ApoE intensity in nucleus and cytoplasm was quantified for untreated and HDAC6 ZnF UBP treated neuro2a immunostained with anti-ApoE antibody. There was no significant difference in cytoplasmic level of ApoE in both groups while the nuclear ApoE fraction was notably increased in HDAC6 ZnF UBP treated cells. C) Enhanced ApoE localization upon HDAC6 ZnF UBP treatment may be mediated by nucleolin dependent transport of ApoE. ApoE requires an active transport system as it contains weak NLS signal. Nucleolin may serve this function as it is a known interacting partner of apolipoproteins and is stabilized by HSP90 whose activity is regulated by HDAC6. D) Neuro2a treated with HDAC6 ZnF UBP shows localization of tubulin focused over the nuclear periphery compared to untreated neuro2a where tubulin is evenly distributed in cytoplasm. Orthogonal projection image shows tubulin localization in nuclear periphery in HDAC6 ZnF UBP treated neuro2a cells. 
Figure 8

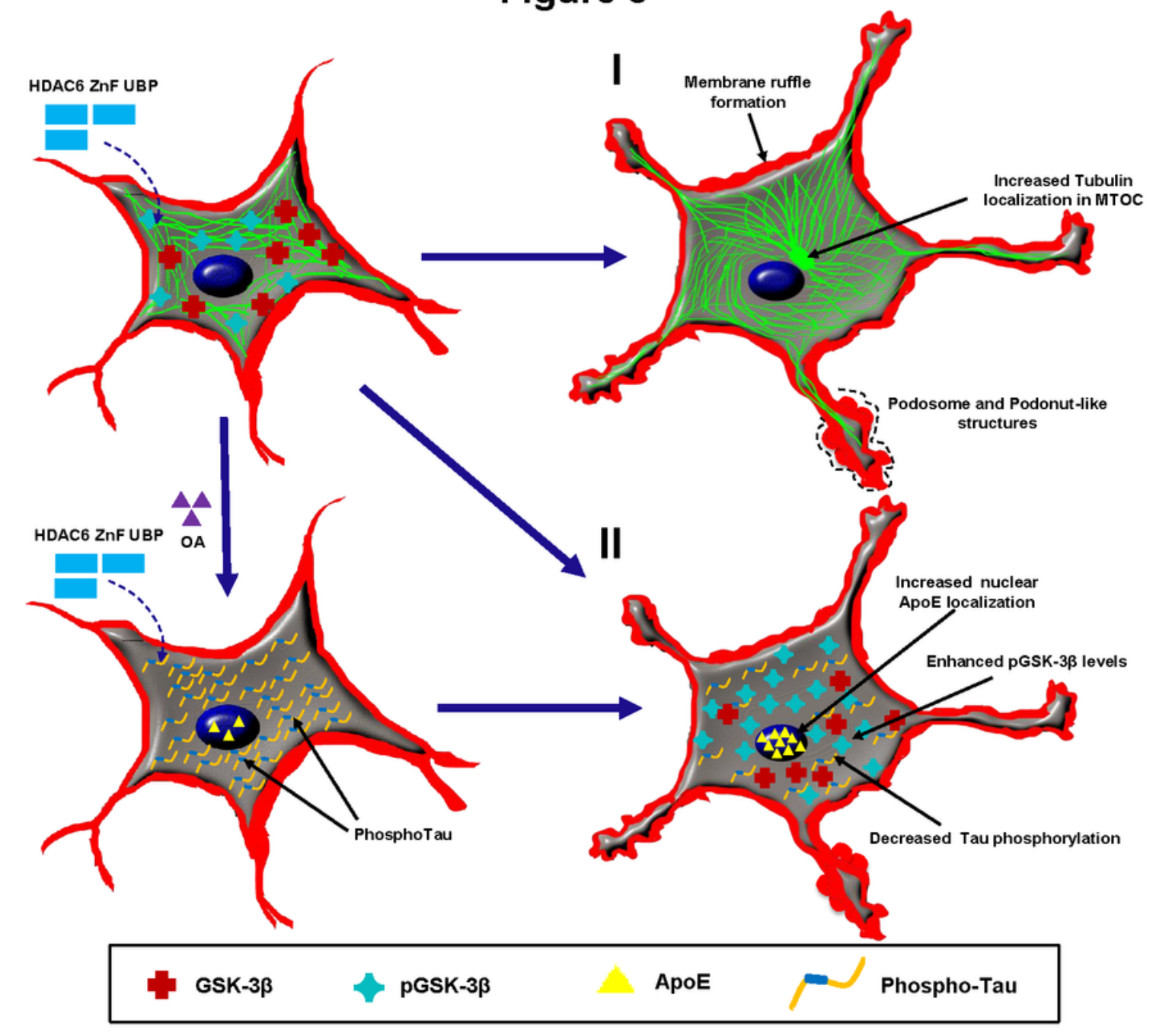

\section{Figure 8}

Effect of HDAC6 ZnF UBP on various cellular functions. HDAC6 ZnF UBP domain was observed to play possible roles in different aspects of neuronal cell function and morphology. Broadly classifying, we observed their effect in remodelling actin and tubulin as well as regulating Tau phosphorylation and GSK$3 \beta$ activity. I) HDAC6 ZnF UBP treatment to the cells resulted in formation of podosome or lamellipodialike structures and enhanced neurite extension. On the other hand, tubulin was found to more localized in 
MTOC in HDAC6 treated cells while untreated cells showed uniform tubulin distribution. II) The phosphorylation of Tau was also found to be reduced when HDAC6 ZnF UBP was given to the cells along with $O A$ as inducer of phosphorylation. OA treated cells showed increased PhosphoTau levels (pT181 and AT8) while the levels of both were reduced in treated cells. The level of pGSK-3 $\beta$ was found to be increased with HDAC6 ZnF UBP treatment while total GSK-3 $\beta$ remains unaltered. Another aspect of HDAC6 treatment was observed in the localization of ApoE. Enhanced nuclear localization was found in treated cells suggesting the role of HDAC6 in regulating ApoE-mediated functions.

\section{Supplementary Files}

This is a list of supplementary files associated with this preprint. Click to download.

- SuppleHDAC6.pdf 